Adick, Christel

\title{
Ein Modell zur didaktischen Strukturierung des globalen Lernens
}

Bildung und Erziehung 55 (2002) 4, S. 397-416

Quellenangabe/ Reference:

Adick, Christel: Ein Modell zur didaktischen Strukturierung des globalen Lernens - In: Bildung und Erziehung 55 (2002) 4, S. 397-416 - URN: urn:nbn:de:0111-pedocs-148195 - DOI: 10.25656/01:14819

https://nbn-resolving.org/urn:nbn:de:0111-pedocs-148195

https://doi.org/10.25656/01:14819

\section{Nutzungsbedingungen}

Gewährt wird ein nicht exklusives, nicht übertragbares, persönliches und beschränktes Recht auf Nutzung dieses Dokuments. Dieses Dokument ist ausschließlich für den persönlichen, nicht-kommerziellen Gebrauch bestimmt. Die Nutzung stellt keine Übertragung des Eigentumsrechts an diesem Dokument dar und gilt vorbehaltlich der folgenden Einschränkungen: Auf sämtlichen Kopien dieses Dokuments müssen alle Urheberrechtshinweise und sonstigen Hinweise auf gesetzlichen Schutz beibehalten werden. Sie dürfen dieses Dokument nicht in irgendeiner Weise abändern, noch dürfen Sie dieses Dokument für öffentliche oder kommerzielle Zwecke vervielfältigen, öffentlich ausstellen, aufführen, vertreiben oder anderweitig nutzen.

Mit der Verwendung dieses Dokuments erkennen Sie die Nutzungsbedingungen an.

\section{Terms of use}

We grant a non-exclusive, non-transferable, individual and limited right to using this document.

This document is solely intended for your personal, non-commercial use. Use of this document does not include any transfer of property rights and it is conditional to the following limitations: All of the copies of this documents must retain all copyright information and other information regarding legal protection. You are not allowed to alter this document in any way, to copy it for public or commercial purposes, to exhibit the document in public, to perform, distribute or otherwise use the document in public.

By using this particular document, you accept the above-stated conditions of use.

\section{Kontakt / Contact:}

peDOCS

DIPF | Leibniz-Institut für Bildungsforschung und Bildungsinformation Informationszentrum (IZ) Bildung

E-Mail: pedocs@dipf.de

Internet: www.pedocs.de

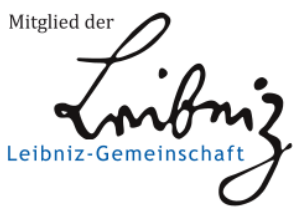




\section{Bildung und

\section{EIN MODELL ZUR DIDAKTISCHEN STRUKTURIERUNG DES GLOBALEN LERNENS.}

Summary: In the last decade, globalisation has reached education in multiple ways, among which is the challenge to teach 'global education' in schools. In the article global education is conceived as a response to - rather than a pure reaction to or a salvation of - globalisation. It is discussed, how global education enters the school: from global via national levels to the levels of subject matter and didactics, and ultimately to classroom activities. In the following some teaching guidelines for global education are referred to from which a new model is constructed. This model, visualised in the form of a 'didactic cube", caters for the critical diagnosis and the constructive didactical analysis of global education.

In den letzten Jahren hat der Globalisierungsdiskurs weltweit auch die Erziehungswissenschaft und ihre Subdisziplinen erfasst (vgl. z.B. ScHEUNPFLUG/HIRSCH [Hg.] 2000, StromQuist/MonKman [Hg.] 2000). Das Thema tangiert im Prinzip pädagogische Fragen unterschiedlichster Art, von vergleichenden Analysen (Auswirkungen von Globalisierung auf nationale Bildungssysteme) über historische Fragestellungen (Genese, Kontinuitäten oder Diskontinuitäten internationaler Bildungsentwicklungen), den Geschlechterdiskurs (Implikationen von Globalisierung für geschlechtergerechte Sozialisations- und Bildungsprozesse), die Bildungsökonomie und -planung (bildungspolitische Optionen angesichts von Globalisierung) bis hin zu didaktischen Fragen (die unterrichtliche Bearbeitung von Globalisierung).

Aus diesem breiten Spektrum soll im folgenden das Augenmerk auf die unterrichtliche Bearbeitung von Globalisierung gelegt werden. Leitend hierfür ist die dezidierte und formelhaft verdichtete Grundannahme, dass globales Lernen als didaktische Antwort auf Globalisierung gesehen wird. Um diese Aussage näher zu erläutern, müssen zunächst die zentralen Begriffe umrissen werden: Was wird hier unter ,Globalisierung ' verstanden? Was ist mit ,globalem Lernen' gemeint? Und was bedeutet es, wenn globales Lernen als ,Antwort' auf Globalisierung gesehen wird? Danach wird die Frage verfolgt, wie globales Lernen in den Unterricht kommt. Hierzu wird der didaktische Diskurs mit Bezug auf unterschiedliche Ebenen in den Blick genommen und an einem Beispiel - der Implementation von ,Bil- 
dung für nachhaltige Entwicklung - illustriert. Hieran schließt sich die Herleitung und Begründung eines didaktischen Modells an, das der Strukturierung des globalen Lernens zum Zwecke des Unterrichts dienen soll. Es wird ein , didaktischer Würfel " vorgestellt, dessen Mehrdimensionalität die Kriterien versinnbildlicht, die für die Planung eines solchen Unterricht maßgeblich sein sollten.

\section{Globales Lernen als Antwort auf Globalisierung}

\subsection{Globalisierung}

Über Globalisierung ist in den letzten Jahren auch im deutschen Sprachraum so viel geschrieben worden (vgl. z.B. die nach Themen gegliederte Bibliographie in BuTTER-WEGGE/HENTGES [Hg.] 2002, S. 311-318), dass hier ein kurzer Blick auf die Hauptbereiche Ökonomie, Politik und Kultur genügen soll: Das Thema betrifft an erster Stelle wirtschaftliche Entwicklungen. Globalisierung bedeutet hier, dass sich Kapitalzirkulation, Produktions- und Vermarktungsprozesse zunehmend auf einem Weltmarkt abspielen, der die staatlich reglementierten Binnenmärkte und die lokal agierenden Privatunternehmen zwar nicht außer Kraft gesetzt hat, aber dennoch deren Existenz erheblich beeinflusst. Neben oder über die volkswirtschaftliche und die betriebswirtschaftliche tritt zunehmend die weltwirtschaftliche Handlungs- und Analyseebene. In politischer Hinsicht haben sich in den letzten Jahrzehnten ebenfalls die Einflüsse weltpolitisch bedeutsamer supranationaler Organisationen (z.B. die Vereinten Nationen und ihre Unterabteilungen) neben den staatlichen, den makro- und mikroregionalen sowie den lokalen politischen Ebenen fest etabliert. Dennoch ist es zwar zu Forderungen nach global governance, aber noch zu keiner Weltregierung gekommen. In Bezug auf den Kulturbereich scheint es ebenfalls fraglich, ob wir wirklich - jenseits der Frage ihrer Wünschbarkeit - von einer ,Weltkultur' reden können; denn die in den letzten Jahren wieder aufkommenden Fragmentierungen als Kehrseite der Globalisierung (MENZEL 1998), wie z.B. ReEthnisierungen, die Gründung neuer Nationalstaaten und die Suche nach eigenen kulturellen Wurzeln und Selbstdeutungen scheinen darauf hinzuweisen, dass die Menschen sich keinesfalls alle und zuerst als Weltbürger sehen mit einem Bekenntnis zu weltkulturellen Gemeinsamkeiten. Andererseits zeigen sich dennoch gerade im Freizeit- und Konsumverhalten von Jugendlichen weltweit ähnliche Verhaltensmuster, und viele Bereiche von Kommunikation, Wissenschaft und Technologie und nicht zuletzt die strukturell und curricular weltweit ähnlichen öffentlichen Bildungssysteme (vgl. ADICK 1995) - werden derzeit schon auf Weltebene praktiziert, so dass man in ihnen Anzeichen für die Entstehung einer Weltkultur sehen kann.

\subsection{Globales Lernen}

Eine eindeutige Definition dessen, was unter ,globalem Lernen " zu verstehen ist. ist ebenso schwierig wie Definitionen und Abgrenzungen zu anderen Ansätzen aus dem internationalen und interkulturellen Bereich von Erziehung und Bildung. wie beispielsweise Friedenserziehung, interkulturelles Lernen, entwicklungspolitische Bildung, Erziehung zur Völkerverständigung, Menschenrechtserziehung oder Bildung für nachhaltige Entwicklung. Erschwerend kommt hinzu, dass in den genannten Begriffen wahlweise und ohne Erkennbarkeit einer Konsistenz oder Logik von ,Lernen', ,Erziehung oder ,Bildung' die Rede ist. Warum hat sich die Rede von interkulturellem Lernen oder interkultureller Erziehung - nicht aber interkultureller Bildung - durchgesetzt? Warum gibt es keinen Begriff wie ,Umwelt-Lernen'? oder ,Friedensbildung'? Warum hat sich im anglophonen Sprachraum der Begriff ,global education' durchgesetzt, im deutschen hingegen der des ,globalen Lernens'?

Da es nicht Anliegen dieses Beitrags ist, eine Genealogie und Abgrenzung des Diskurses um ,globales Lernen “ vorzulegen, sondern didaktische Strukturierungskriterien zu entwerfen, begnügen wir uns hier mit der Entscheidung, ,globales Lernen' als einen Oberbegriff für all jene didaktischen Bemühungen zu sehen, die sich auf die Implementation und Strukturierung solcher Unterrichtsgegenstände richten, deren Fokus die gesamtmenschheitliche Lebenspraxis darstellt. Curricula und Unterricht in einem nationalstaatlich organisierten öffentlichen Schulsystem haben demzufolge - emphatisch pointiert - nicht mehr oder jedenfalls nicht mehr ausschließlich die Tradierung (vermeintlicher oder realer) ,nationaler Kulturguiter zum Ziel, sondern müssen (auch) einen Beitrag zur Heranbildung zukünftiger Weltbürger leisten.

Globales Lernen steht also als Oberbegriff und integriert damit verschiedene, teils schon ältere Ansätze, die sich mit weltbürgerlichen Perspektiven beschäftigen. Als solche nennt etwa KENNETH A. TYE, der in den 1990er Jahren eine weltweite Umfrage zu dem Thema durchgeführt hat, die folgenden: peace education, environmental education, intercultural education. development education, human rights education, education for democracy, human geography, world studies (TYE 1999, Kap. 8). DAvID SELBY, Direktor des International Institute for Global Education an der Universität von Toronto, spricht hingegen von folgenden Schlüsselbereichen des globalen Lernens: development education, environmental education, human rights education, peace education, health education, gender equity education, education for a multicultural societs; humane education, citizenship education, media education (SELBY 2000, Tab. 1, S. 5). 
Wenngleich die Schnittmenge zwischen beiden Autoren erheblich ist, so zeigen sich doch etliche Unterschiede. Zum relativ unstrittigen Kernbereich des globalen Lernens würden demnach Friedenserziehung, interkulturelles Lernen, Menschenrechtserziehung, entwicklungspolitische Bildung und Umweltbildung zählen. Zu bedenken ist jedoch, ob nach dem Motto, dass schließlich alles mit allem zusammenhängt, mit der Nennung von Bereichen wie Humangeographie, staatsbürgerliche Bildung oder Medienerziehung der Begriff des globalen Lernens nicht über Gebühr strapaziert wird und damit an Kontur verliert. Aus diesem Grunde wird hier die Auffassung vertreten, es zumindest vorerst bei der oben genannten Schnittmenge' zu belassen. Orientierungspunkt für diese Entscheidung ist der schon angeführte Bezug auf die gesamtmenschheitliche Lebenspraxis statt auf eine partikulare, beispielsweise eine nationale Tradition.

Zugleich soll der Begriff ,globales Lernen' darauf aufmerksam machen, dass die betreffenden Unterrichtsgegenstände ,global', d.h. ganzheitlich, multiperspektivisch und umfassend in den Blick genommen werden sollen und nicht in einer fragmentierten, partikularen oder disziplinspezifisch verkürzten Art und Weise. Unterstrichen wird dieser Gesichtspunkt im deutschen Kontext etwa dadurch, dass entsprechende Unterrichtsgegenstände immer als Teil der Allgemeinbildung, nicht hingegen als neues Unterrichtsfach oder als bloß additiv hinzu kommendes Thema begriffen werden, wie es etwa die entsprechenden KMKEmpfehlungen zur Menschenrechtserziehung (KMK 1980/2000), zur Interkulturellen Bildung und Erziehung in der Schule (KMK 1996) oder zu Eine Welt/ Dritte Welt in Unterricht und Schule (KMK 1997) deutlich machen. Auch im internationalen Vergleich zeigt sich, dass etliche Bildungssysteme Ziele wie interkulturelles Lernen in ihren Allgemeinbildungsanspruch aufgenommen haben (vgl. z.B. DAvies/GuPPY 1997, S. 442 ff.), so dass auch in der dortigen Schulpraxis der integrative (statt additive) Weg der curricularen Verankerung gewählt wird. Auch die diversen UNESCO-Empfehlungen bspw. zur Erziehung zur internationalen Verständigung und zur Menschenrechtserziehung (UNESCO 1974, 1998) gehen diesen Weg.

\subsection{Globales Lernen als Antwort auf Globalisierung}

In der schon genannten, in den 1990er Jahren durchgeführten international vergleichenden Untersuchung zur Konzeption und Praxis globalen Lernens in 52 Ländern (TYE 1999) wurde den Befragten folgende dem Jahrbuch 1991 der US-amerikanischen Association for Supervision and Curriculum Development entnommene Definition von global education zur Stellungnahme vorgelegt:
Global education involves learning about those problems and issues which cut across national boundaries and about the interconnectedness of systems cultural, ecological, economic, political, and technological ...

Global education also involves learning to understand and appreciate our neighbors who have different cultural backgrounds from ours; to see the world through the eyes and minds of others; and to realize that other people of the world need and want much the same things." (zit. in TYE 1999, S. Xv).

Wenngleich positiv zu vermerken ist, dass die Definition sowohl internationale wie auch interkulturelle Aspekte verbindet, so hebt sie dennoch zu wenig ab auf die produktive Auseinandersetzung der Lernenden mit ,Globalisierung'. Wenn hier globales Lernen als ,Antwort' und nicht etwa als ,Reaktion' auf Globalisierung bezeichnet wird, so soll dies darauf hindeuten, dass damit keine bloße Inkorporation weltgesellschaftlicher Daten und Fakten in den Unterricht (learning about those problems and issues which cut across national boundaries ...) und auch kein bloßer Perspektivenwechsel zwischen Eigenem und Fremdem (learning ... to see the world through the eyes and minds of others...) gemeint ist, sondern dass es hier um in ihrer Art letztlich auch pädagogisch nicht determinierbare, weil subjektive Aneignungsleistungen, um ,Antworten“ im Sinne kritischer Auseinandersetzung und produktiver Bearbeitung geht. Denn gerade die Schule, in der sich Lehrende und Lernende konstruktiv Wissen und Kultur aneignen, ist auch an der möglichen Entstehung eines kritischen Potentials und neuer Ideen durch eine aktive Auseinandersetzung mit der sich entwickelnden Weltgesellschaft beteiligt (vgl. ADICK 2000, S. 159). Eine solche Vorstellung von Unterricht in einem öffentlichen Schulwesen und von globalem Lernen als Teil der Allgemeinbildung begreift sich gerade deshalb nicht als bloße Affirmation weltgesellschaftlicher Bedingungen, sondern als kritische Aufarbeitung derselben: „Globales Lernen möchte Menschen dazu befähigen und dazu ermutigen, an der Gestaltung der Weltgesellschaft sachkundig und verantwortungsbewusst teilzuhaben. Es zielt auf eine Form des Lernens und eine Weise des Denkens, die es erlauben, lokale Gegebenheiten in ihrer Einbindung in den globalen Kontext wahrzunehmen, und dazu zu befähigen, lokales Handeln in Einklang mit globalen Erfordernissen zu bringen." (SEITZ 2002, S. 50).

Dabei muß jedoch bedacht werden, daß globales Lernen auch nicht als ,Lösung ' der Globalisierungsprobleme mißverstanden werden darf, ,, hat sich doch die Welt nicht in gleichem Maße friedlicher, toleranter und gerechter entwickelt, in dem diese Ziele im Unterricht thematisiert wurden“, wie Traugott Schöfthaler (2000, S. 21) zur internationalen Entstehungsgeschichte des globalen Lernens schreibt. Als pädagogische ,Antwort' auf Globalisierung kann globales Lernen 
sich nur auf die Zielebene des Handlungspotentials ausrichten: „Es erscheint einleuchtend, dass die Fähigkeit zum Perspektivenwechsel, zur Multiperspektivität, zur Akzeptanz von oder gar Neugier auf Meinungen, Werte und Traditionen anderer Menschen, die sich mit den Zielen Pluralismus und Vielfalt verbindet, mehr Handlungspotential erschließt als reine Wissensakkumulation." (ebd.).

\section{Globales Lernen als Gegenstand makro- und mikrodidaktischer} Entscheidungen

Programme und Konzeptionen des globalen Lernens sind keinesfalls mit ihrer unterrichtlichen Vermittlung und noch weniger mit dem (erhofften) Lernergebnis der Schülerinnen und Schüler zu verwechseln. Inhalte und Ziele eines Unterrichts, der sich auf ,globales Lernen ' ausrichtet, gelangen weder unvermittelt in die Schule noch in die Köpfe der Schüler. Aus diesem Grunde soll im folgenden verfolgt werden, auf welchen verschiedenen Ebenen mit unterschiedlichen Akteuren und Diskursen didaktische Entscheidungen anzutreffen sind, die den Weg der Umsetzung einer globalen Programmatik bis in die spezifische Unterrichtssituation kennzeichnen. Hierzu muss eine kurze Klärung des verwendeten Didaktik-Begriffs vorweg geschickt werden, ohne jedoch auf verschiedene didaktische Traditionen näher eingehen zu können.

Die Didaktik beschäftigt sich mit der Frage, wie aus einem, Thema' ein pädagogisch begründeter und vorgeplanter ,Unterrichtsgegenstand ' wird, ein Gegenstand, der durch Lehrende und Lernende unterrichtlich konstituiert und bearbeitet wird. Damit schließt dieser weite Didaktikbegriff den Begriff der Methodik mit ein. Er besagt zudem, dass didaktische Planung auf Unterricht ausgerichtet ist, sich also nicht auf eine Theorie der Lehrinhalte beschränken kann, und dass didaktische Planung Unterricht wohl vorstrukturieren und in Grenzen antizipieren, aber wegen der Eigentätigkeit der Lernenden nicht vollkommen determinieren kann.

Der Zweck des Unterrichts in der öffentlichen Pflichtschule besteht - formelhaft verdichtet - in folgendem: ,Im Unterricht sollen die Heranwachsenden Möglichkeiten der Menschheit als ihre eigenen Möglichkeiten erkennen und sich zu eigen machen." (AdiCK/Bonne/MENCK 1978, S. 187). In dieser Formel verbirgt sich die Tatsache, dass aus dem Universum möglichen menschlichen Wissens eine Auswahl getroffen werden kann und muss, wie auch das pädagogische Anliegen, die Heranwachsenden möchten sich - potentielles Scheitern inbegriffen - mit diesen ausgewählten menschlichen Wissensbeständen aktiv und selbstaneignend beschäftigen. Die gewählte Definition ist, obschon damals nicht für diesen Zweck geschrieben, auch kompatibel mit den Anliegen des ,globalem Lernens", da in ihr nicht von der Tradierung bestimmter Kulturgüter die Rede ist. sondern von der Heranführung an ,Möglichkeiten der Menschheit', um partikularistische Engführungen zu vermeiden.

Wie werden nun aber, Themen ‘ zu ,Unterrichtsgegenständen ' bzw. wie kommt ein Thema in den Unterricht? Hier ist zwischen verschiedenen „Bezugsebenen didaktischer Reflexion“, wie Peter Menck sie in Anlehnung an HeRwiG BLANKERTZ bezeichnet, zu unterscheiden: 1. das didaktische Gesamtsystem, 2. die Fachdidaktik, 3. die didaktische Analyse, 4. die Didaktik der Unterrichtssituation oder Mikrodidaktik (MENCK 1975, S. 36 ff). Ähnliche Überlegungen finden sich auch bei anderen Autoren. So sprechen z.B. PETER POSCH u.a. (1996, S. 187 ff.) von fünf verschiedenen „Ebenen der Konkretisierung bildungspolitischer Leitideen“: 1. das Schulsystem, 2. die Schulart, 3. der Unterrichtsgegenstand. 4. die Konzeption des Unterrichts, 5. die Unterrichtsrealität.

Trotz der Unterschiede nehmen beide Modelle ihren Ausgang bei dem jeweiligen Bildungssystem als Ganzem, das z.B. Schulstrukturen und Lehrpläne verordnet und vorgibt, und enden in der tatsächlichen Unterrichtspraxis. in der ein Thema in den Interaktionen und Handlungen von Lehrpersonen und Lernenden Gestalt annimmt. Beiden Modellen gemeinsam ist indessen auch, dass sie die oberste Ebene des Gesamtsystems als eine nationale konzipieren; eine der nationalen über- oder vorgelagerte, globale' didaktische Bezugsebene fehlt indessen. Diese den Ebenen hinzuzufügen ist jedoch angesichts der Entstehung eines ,Weltmodells Schule' mit transnationalen Merkmalen und globalen Akteuren, die auf die Ebene der einzelstaatlichen Bildungspolitik Einfluss ausüben, unumgänglich '. Sie ist ferner konstitutiv, wenn man, wie hier vorgeschlagen, globales Lernen als Antwort auf Globalisierung begreifen will. Makrodidaktische Überlegungen müssen demnach im Zeitalter der Globalisierung bereits bei der Weltebene und nicht erst bei den jeweiligen nationalen Bildungssystemen ansetzen.

\subsection{Von makro- zu mikrodidaktischen Entscheidungen}

Aus diesen Vorstellungen ergibt sich folgendes makro- bis mikrodidaktische Denkmodell (Tab. 1), das verdeutlichen will, auf welchem Wege, Themen' uiber verschiedene Bezugsebenen in den Unterricht kommen (können) und in welcher Gestalt (Diskurse) sie didaktisch gehandhabt werden. Da die Anordnung der Ebenen von der ersten (globalen) bis zur letzten (mikrodidaktischen) einen unidirektionalen Ableitungszusammenhang suggerieren mag, sei hervorgehoben, dass die verschiedenen Ebenen als miteinander verflochten gedacht werden. was Riickkopplungseffekte und Wechselwirkungen einschließt. 


\begin{tabular}{ll}
\hline 1. Weltmodell Schule & $\begin{array}{l}\text { Aktionen und Programme supra- und } \\
\text { internationaler Organisationen, z.B. } \\
\text { UNESCO, OECD; internationale } \\
\text { pädagogische Fach- und Interessenver- } \\
\text { bände }\end{array}$ \\
\hline 2. Nationales Schulsystem & $\begin{array}{l}\text { rechtliche und bildungspolitische } \\
\text { Vorgaben, Lehrpläne, Prüfungs- und } \\
\text { Berechtigungswesen }\end{array}$ \\
\hline 3. Unterrichtsfächer & $\begin{array}{l}\text { Fachdidaktiken, Lehrpläne, Schulbücher, } \\
\text { teils nach Schulform und Schultyp, } \\
\text { fachübergreifende/ interdisziplinäre } \\
\text { didaktische Konzepte }\end{array}$ \\
\hline 4. Unterrichtsgegenstand & $\begin{array}{l}\text { didaktische Analyse und Unterrichts- } \\
\text { planung einzelner Unterrichtsreihen/ } \\
\text { Themen }\end{array}$ \\
\hline 5. Unterrichtspraxis & unterrichtliche Interaktionen, Lehr- und \\
& Lernprozesse \\
\hline
\end{tabular}

Tab. 1: Makro- und mikrodidaktische Ebenen

Wie werden diesem Denkmodell zufolge Themen globalen Lernens zu Unterrichtsgegenständen? Welche Rolle spielen die verschiedenen Ebenen und was steuern sie zum didaktischen Diskurs bei? Sind sie miteinander vernetzt und beziehen sich die Diskurse aufeinander? Dies soll am Beispiel der Implementation des jüngsten Ansatzes des globalen Lernens: Bildung für eine nachhaltige Entwicklung, durchgespielt werden.

2.2 Bildung für nachhaltige Entwicklung: der Weg von der globalen

Programmatik zur Unterrichtspraxis

Ausgangspunkt auf der globalen Ebene war hier die Verabschiedung der Agenda 21 der Konferenz der Vereinten Nationen über Umwelt und Entwicklung im Jahre 1992 in Rio. In Kap. 36 dieser Agenda (u.a. abgedruckt in BLK 1998) werden Erziehung, Bewusstseinsbildung und Ausbildung als Schlüsselvariablen zur Verwirklichung des Anliegens der nachhaltigen Entwicklung gesehen. In der Bundesrepublik wird dazu eigens im August 1999 ein auf fün Jahre ausgelegtes und mit einem Gesamtetat von fast 13 Mio. Euro finanziell recht gut ausgestattetes Programmpaket zur Umsetzung in den schulischen Kontext gestartet (vgl, www.blk21.de). Nachdrücklich unterstützt wird dieses Vorhaben durch eine Empfehlung des Deutschen Bundestages (Drucksache 14/3319) im Jahre 2000. Bildung für eine nachhaltige Entwicklung, insbesondere in ihren zwei Hauptsäulen der Umweltbildung und der entwicklungspolitischen Bildung. als neues Leitziel in die bestehenden Bildungsstrukturen zu implementieren. Inzwischen entstehen viele lokale, regionale oder deutschlandweite Initiativen, die sich dieses Anliegens annehmen, entsprechende Projekte ins Leben rufen, Unterrichtsmaterialien verfassen, Zeitungen gründen, Kongresse durchführen und Publikationen auf den Weg bringen. Als Beispiele aus dem breiten Arsenal dieser Initiativen verschiedenster Akteure seien nur folgende genannt:

Das BLK 21 Programm koordiniert ein bundesweites Netzwerk von Modellschulen und unterhält Ansprechpartner in den einzelnen Bundesländern. Es gibt mit der Nullnummer im September 2000 eine eigene Zeitschrift heraus: ,21 - Das Leben gestalten", in der sich dann bald auch didaktische Leitartikel finden (de Haan/Seitz 2001); es unterhält einen Lenkungsausschuss, einen wissenschaftlichen Beirat und eine Arbeitsgruppe Evaluation. Es entwickelt eine Grundstruktur didaktischer Leitlinien in Form von drei Modulen mit den Titeln: interdisziplinäres Wissen, partizipatives Lernen und innovative Strukturen, gibt entsprechende Materialien heraus und veranstaltet bereits im Juni 2001 den ersten Kongress. auf dem Bildungsakteure aus allen Bereichen des Bildungswesens ihre Ideen und Produkte vorstellen, von denen dann 66 Beispiele als best practice auf einer CD-ROM verbreitet werden (BLK 2001).

Auch Nicht-Regierungsorganisationen sind an dem sich konstituierenden didaktischen Diskurs beteiligt. So gibt z.B. das Deutsche Komitee des World University Service (WUS) eine Publikation heraus, die sich der Förderung des globalen Lernens/der Bildung für nachhaltige Entwicklung widmet und hierzu die Institutionen. Gesetzesgrundlagen, ferner die Lehrplan- und Schulbuchsituation sowie Projekte und Ansätze in den verschiedenen deutschen Bundesländern zusammenstellt (WUS 1998). Erhebliche Breitenwirkung dürfte außerdem ein Kongress gehabt haben. den der Verband Entwicklungspolitik deutscher Nicht-Regierungsorganisationen (VENRO), der sich als Dachverband auch mit der entwicklungspolitischen Bildungsarbeit in Deutschland beschäftigt, im September 2000 in Bonn zum Thema „Bildung 21 - Lernen für eine gerechte und zukunftsfähige Entwicklung“ durchführte. Zu dieser vom Bundesministerium für wirtschaftliche Zusammenarbeit und Entwicklung (BMZ) und von den Kultusministerien unterstützten Tagung kamen immerhin ca. 700 Teilnehmer aus allen Bereichen der Bildungspraxis. Wissenschaft, Verwaltung und Politik. Die Abschlusserklärung und die Dokumentation des Kongresses machen deutlich, daß Bildung für eine nachhaltige Entwicklung keinesfalls auf Umweltbildung oder die Stärkung einer auf Zukunft ausgerichteten 
internationalen Wettbewerbsfähigkeit reduziert werden darf, sondern in globaler Perspektive die Fortführung der Ziele des interkulturellen und des entwicklungsbezogenen Lernens umgreifen muss (VENRO 2001).

In diesem Beispiel ist der von der globalen Ebene (Rio-Konferenz 1992) angestoßene didaktische Diskurs zwar mit einiger zeitlicher Verzögerung, aber dann doch deutlich von der Ebene des nationalen, in diesem Falle des deutschen Bildungswesens aufgegriffen worden. Der didaktische Diskurs spielt sich zwischen staatlichen und nicht-staatlichen Bildungsakteuren überwiegend auf der nationalen Ebene ab, greifbar z.B. in den bundesweiten Kongressen. Er hat aber auch schon die Ebene der didaktischen Analyse und die Unterrichtspraxis erreicht, wie z.B. die best practice Beispiele des BLK-Kongresses 2001 zeigen. Weniger betroffen vom Diskurs um Bildung für nachhaltige Entwicklung scheint bisher allerdings die fachdidaktische Ebene zu sein, deren Diskurs überwiegend in Gestalt von Lehrplänen und Schulbüchern greifbar ist. Das mag einerseits daran liegen, dass Bildung für nachhaltige Entwicklung als Querschnittsthema definiert ist, so dass sich die Fachdidaktiken hiervon weniger angesprochen fühlen. Andererseits ist dieser Bereich gerade in Deutschland mit seinen recht ,behäbigen ' Curriculumprozeduren in Form von länderspezifischen Lehrplankommissionen und Schulbuchgenehmigungen geradezu zu einem größeren time lag prädestiniert als die anderen Ebenen.

Nachdem somit beschrieben ist, auf welchen Ebenen, Themen ' konstituiert (und gefiltert, modelliert) werden und dann in den Unterricht kommen und von dort eventuell wieder zurück zu Lehrplankommissionen usw. (was aus Platzgründen hier nicht weiter diskutiert werden kann), soll nun gefragt werden, wie diese ,Themen' für die Unterrichtsplanung didaktisch strukturiert werden können. Denn bei der didaktischen Strukturierung globalen Lernens ist es nicht damit getan, eine Reihe von Zielen oder eine Liste von Inhalten, ggf. fach- und altersspezifisch sortiert, zu benennen. Es muss auch begründet werden, mit welcher inhaltlich-intentionalen Ausrichtung der Bildungserwerb gesteuert werden und welche Art von Aneignungsprozeß auf Seiten der Lernenden stattfinden soll

3. Die didaktische Strukturierung des globalen Lernens zum Zwecke der Unterrichtsplanung

Im folgenden wird ein dreidimensionales didaktisches Modell in Gestalt eines ,didaktischen Würfels' vorgeschlagen, das auf den Konzeptionen von DAviD Selby (2000) und Peter Weinbrenner/K.-Peter Fritzsche (1993) aufbaut und diese weiterentwickelt. Bevor im Anschluß das eigene Modell erörtert wird, sollen zuvor diese beiden Konzeptionen kurz vorgestellt werden ${ }^{2}$
3.1 Die Ansätze von Selby und Weinbrenner/Fritzsche

David Selby schlägt ein vierdimensionales Modell globalen Lernens vor. .reflecting the global educator's twin and complementary goals of helping students explore the dynamics, condition and future of the world in which they live (the ,global village') and through that exploration, helping them better comprehend. realise and utilise their own potential as human beings (the ,global self"). All four dimensions are to be seen as profoundly interrelated." (SELBY 2000. S. 2 f.).

Die von ihm konzipierten Dimensionen sind die folgenden (vgl. ebd., S. 3 ff.):

- Die räumliche Dimension (.The Spatial Dimension“) tangiert alle Ebenen vom einzelnen Individuum bis zum ganzen Globus. Die Skala wird unterteilt in: intrapersonal, interpersonal, lokal, bioregional, national, international und global. Die Ebenen werden jedoch nicht als konzentrische Kreise vom Nahen zum Fernen aufgefasst, sondern stellen eine Einheit (an , unbroken wholeness') dar.

- Die thematische Dimension (,The Issues Dimension") umfasst die Schluisselthemen des globalen Lernens, wie sie im obigen Zitat von SELBY (vgl. Abschnitt 1.2.) schon genannt wurden.

- Die Zeitdimension (,The Temporal Dimension") umfasst Vergangenheit, Gegenwart und Zukunft, wobei Selby betont, dass die pädagogische Intentionalität sich insbesondere auf die Zukunft ausrichten solle, indem im Unterricht alternative Szenarien (,possible', ,probable ' and ,preferred' futures) reflektiert werden sollten. Dabei gehe es nicht so sehr um Prognosen, sondern Zukunft solle als ein Potential noch offener Möglichkeiten gesehen werden. Die Lernenden sollen erkennen, daß menschliches Handeln, einschließlich ihres eigenen, in die Gestaltung der Zukunft einfließt.

- Die vierte Dimension, von Selby ,The Inner Dimension“ genannt, bezieht sich auf das lernende Subjekt als Teil eines holistisch gedachten Weltganzen: „The holistic paradigm emerging from fields such as quantum physics sees the wellbeing and prospects of person and planet as in dynamic synthesis: if each of us endeavours to bring together the many dimensions of our divided self we benefit the planet and if we work towards a better world we emerge with heightened powers and a profounder conception of our own personhood. Relational holisms. Our inner world, therefore, is as much the focus of global education as our outer world. Our programmes and projects need to address the ,global self as much as the ,global village:"“ (ebd., S.8).

Am Ende seiner didaktischen Überlegungen präsentiert der Autor dann noch eine komplexe Übersicht, in der diese vier Dimensionen mit den aus der Didaktik all- 
seits bekannten Lernzieldimensionen Wissen, Fertigkeiten und Einstellungen (,knowledge', ,skills', , attitudes') in Beziehung gesetzt werden (ebd., S. 9).

Das zweite Referenzmodell ist der ,didaktische Würfel` zur Menschenrechtserziehung von WeINBRENNER/FrITZSCHE (1993, S. 31), der laut Angabe der Autoren aus der Evaluation von Lehrbüchern aus verschiedenen Ländern und aus Menschenrechtsdokumenten hervorging. Das Modell des Würfels stellt die Zusammenschau von Reflexionsprozessen verschiedener didaktischer Entscheidungsdimensionen dar. In Bezug auf die Zielebene beziehen sich die Autoren ebenfalls auf die bekannte Unterscheidung zwischen Wissen, Einstellungen und Fertigkeiten (,knowledge', attitudes/values', , skills'; ebd., S. 8). In Bezug auf die Inhaltsebene führte die Suche nach didaktischen Strukturierungskriterien (,didactic principles of selection and legitimation') die Autoren dazu, diese aus dem Menschenrechtsdiskurs heraus zu entwickeln. Hieraus ergab sich eine Stratifizierung entlang einer Linie von individuellen über soziale zu kollektiven und schließlich zu globalen Menschenrechten (ebd., S. 16). Allerdings stellte sich die Frage der Inhalte offenbar insgesamt als etwas sperrig, wenn nicht gar beliebig heraus. Denn die Autoren konstatieren, dass offenbar eine Fülle von Themen unter dem Titel Menschenrechtserziehung im Unterricht behandelt werden könnten, wobei jedoch nicht erkennbar sei, nach welchen Gesichtspunkten (,essentials ') diese unterrichtet werden sollten. Paradoxerweise, wie die Autoren meinen, sei Menschenrechtserziehung offenbar, goal-rich ' and , content-poor', repräsentiere also eher eine bestimmte Perspektive denn einen spezifischen Inhalt (ebd., S. 24).

In der weiteren Argumentation werden dann drei distinkte, aber miteinander verwobene Dimensionen unterschieden, die zunächst in einer Tabelle zusammengefasst und schließlich in Gestalt eines ,didaktischen Würfels" visualisiert werden (ebd., S. 30 f.). Obwohl die Achsen des Würfels leider nicht benannt sind und es im Einzelnen auch nicht ganz nachvollziehbar ist, wie die Autoren zu ihrem Würfelmodell gelangten, versinnbildlichen die drei Achsen offenbar folgende Gedankenstränge:

- Eine Achse repräsentiert mit dem Hinweis auf Vergangenheit, Gegenwart und Zukunft unschwer erkennbar die ,Zeitdimension“

- Eine weitere bezieht sich auf eine Dimension, die ich ,Kontextdimension“ nennen würde, da die Autoren hier zwischen Gesichtspunkten von der individuellen (,my rights', what can I do?) bis zur globalen Ebene (,world', , what can the UN and other world organisations do?) unterscheiden.

- Am schwierigsten ist hingegen die Benennung der dritten Achse; denn auf dieser tauchen drei Perspektiven auf, mit denen über Menschenrechte unterrichtet werden soll: eine normative, die sich mit Menschenrechtsdeklarationen beschäf- tigt (,value approach'), eine empirische, die auf Menschenrechtsverletzungen gerichtet ist (,critical approach') und eine handlungsorientierte, auf die Durchsetzung von Menschenrechten ausgerichtete Perspektive (,political approach'). Man könnte geneigt sein, diese als ,Zieldimension` zu bezeichnen, würde dabei aber die Inhalte unterschlagen, die ebenfalls in den Perspektiven enthalten sind und das Gemeinte damit verkürzen. Es geht hier offenbar nicht nur um Ziele, sondern gleichzeitig um die Struktur des Wissens und die Art der Wissensvermittlung.

Bevor das Problem gerade dieser dritten Dimension didaktischer Strukturierung wieder aufgegriffen wird, sei ein kurzer Vergleich zwischen der Konzeption von SELBY und der von WeINBRENNER/FrITZSCHE eingeschoben. Zwei Dimensionen scheinen nahezu deckungsgleich zu sein: die historische oder Zeitdimension und die Kontextdimension von der individuellen bis zur globalen Ebene, die bei SELBY ,The Spatial Dimension“ heißt. Welche Entsprechung hat aber die Dimension normativ - faktisch - handlungsorientiert' von WEINBRENNER/FRITZSCHE im Konzept von SELBY? Repräsentiert sie die Inhaltsdimension oder die ,Inner Dimension', oder gar beide?

Bei näherem Hinsehen findet sich bei SELBYs letzter Übersicht in seiner Inhaltsdimension eine Unterteilung, die die Inhalte als Zielvorstellungen wendet; denn Inhalte (,issues“) tauchen nun in den Stichworten nur mehr als „,interpersonal/local/global issues; interconnectedness between issues; perspectives on issues; common moral values" auf, die dann weiter nach den Zieldimensionen Wissen, Fertigkeiten und Einstellungen differenziert werden, wobei überhaupt keine konkreten Inhalte mehr auftauchen, sondern immer nur Zielperspektiven (z.B. ,knowledge ... of how perspectives are shaped'), unter denen Wissensbestände angeeignet oder Einstellungen erworben werden sollen (SELBY 2000. S.9). Auf diese Weise sind diese Kriterien aber im Endeffekt genauso, goal-rich and content-poor' geraten, wie dies schon als einigermaßen paradoxe Situation von WEINBRENNER/FRITZSCHE für die Menschenrechtserziehung diagnostiziert wurde.

Ist aus diesem Befund abzuleiten, dass es praktisch unmöglich ist, eine Positivliste von Inhalten des globalen Lernens vorzulegen? Dass es statt dessen nur möglich ist, Perspektiven zu benennen, unter denen entsprechende Themen zu unterrichten wären in dem Sinne, wie WeINBRENNER/FrITZSCHE zu dem Schluss gekommen waren, Menschenrechtserziehung sei eher eine Perspektive denn ein bestimmter Unterrichtsinhalt?

Die Frage betrifft ein Kernproblem der Didaktik, das unhintergehbare Implikationsverhältnis von Inhalten und Zielen. Es ist unmöglich, Ziele ohne Inhalte zu unterrichten und vice versa. Beide bedingen sich gegenseitig und es ist Aufgabe der Didaktik, eine begründete Auswahl und Strukturierung beider Aspekte zugleich 
als einer unauflöslichen Einheit zu treffen. Darauf hatte schon Blankertz in seiner scharfzüngigen Kritik an lernzieltaxonomischen Didaktiken verwiesen (BLANKERTZ 1972, S. 157 ff.). Konstruktiv gewendet wird das Problem von PETER MENCK mit Bezug auf Comenius dergestalt, dass von einem unauflöslichen Zusammenhang von Wissen und Gewissen, von scientia und conscientia ausgegangen wird, mit dem es Unterricht in der Schule zu tun habe (MENCK 2000, S. 96 ff): Hierbei solle conscientia heute nicht mehr, wie dies noch der Didaktik von Comenius zugrunde lag, als ein bestimmter, geschlossener Moralkodex verstanden werden, sondern beziehe sich auf die Urteilskraft und Selbstverantwortlichkeit eines Subjekts für seine Lebensentscheidungen. In der Vermittlung von scientia und conscienta geniige die Schule ihrem Zweck, einen Beitrag zur Mündigkeit der Heranwachsenden zu leisten und ihnen ein eigenverantwortliches Leben in der Welt zu ermöglichen.

Das, was der Unterricht mit scientia und conscientia zu tun hat, steckt in der von den Autoren begrifflich nicht gefassten - Dimension von WEInBRENNER/ FRITZSCHE, die mit Normen, Fakten und Handlungsorientierung zu tun hat, die gleichzeitig aber auch Inhalte enthält, an denen dies gelernt werden soll. Bei SELBY ist beides auseinandergezogen in die thematische und die ,inner dimension ', wobei die thematische Dimension in seinem letzten Schaubild auch schon sehr conscientia-lastig erscheint und die, imner dimension ' sich einem spezifischen, und zwar holistischen Anspruch an die Bildung des Gewissens verschreibt. Dass mit einem solchen ,modernen ' geschlossenen Weltbild die Grundidee wieder dem nahekommt, was Comenius mit seiner an einem christlichen Weltbild orientierten Vorstellung von der Einheit von scientia und conscientia verkörperte, sei hier nur kritisch angemerkt und bedürfte einer eigenen Erörterung.

Unterrichtliches Handeln als Bearbeitung und Aneignung von Wissen und Gewissen bezieht sich also auf die Weitergabe von ,Kultur'. Didaktik hat demnach die Aufgabe, dafür zu sorgen, dass diese Weitergabe von Kultur gelingt, zum Wohle der Lernenden wie auch im Interesse der Reproduktion der Gesellschaft, wobei mit Reproduktion keine identische Kopie kultureller Traditionen gemeint ist, sondern eine aktive Aneignung im Sinne der eingangs vorgebrachten Aussage, dass im Unterricht Heranwachsende Möglichkeiten der Menschheit als ihre eigenen Möglichkeiten erkennen und sich zu eigen machen sollen.

3.2 Das Modell: der, didaktische Würfel' zur Strukturierung des globalen Lernens

In das folgende eigene didaktische Modell gehen eine historische und eine Kontextdimension ein, die mehr oder weniger den beiden referierten Konzeptionen entsprechen. Für die dritte Dimension wurde als Resultat des eben diskutierten inhaltlich-intentionalen Implikationszusammenhangs die Bezeichnung ,Übermittlung von Kultur' gewählt mit der in Anlehnung an MENCK getroffenen Spezifizierung, dass es sich dabei um den Zusammenhang von Wissen und Gewissen handelt. Unterricht soll faktisches Wissen vermitteln, normative Reflexionen anleiten und zu handlungsorientierten Dispositionen bei den Lernenden beitragen. Um den Gedankengang zu veranschaulichen wurde die von WEINBRENNER/FrITZSCHE vorgelegte Idee eines didaktischen Würfelmodells übernommen.

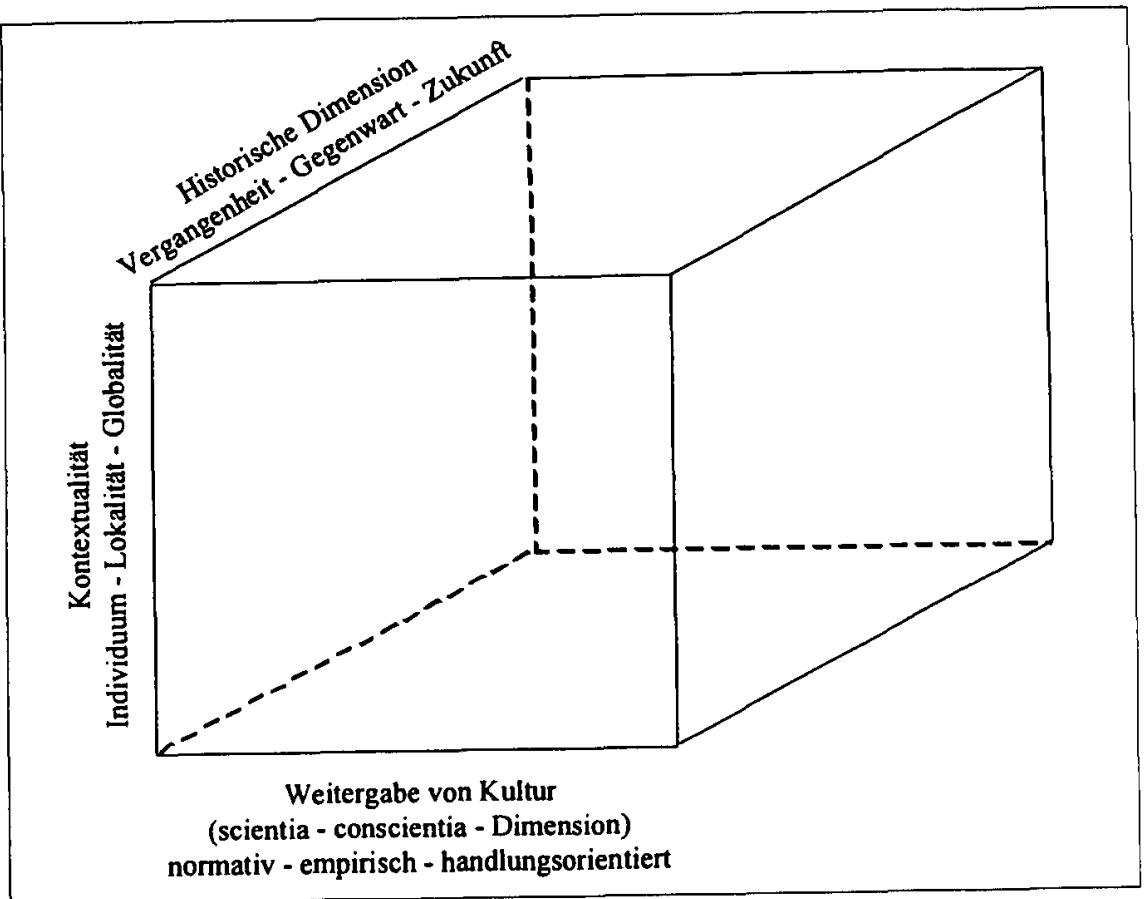

Abb. 1: Der didaktische Würfel zur Strukturierung des globalen Lernens (@ Christel Adick

Die Idee hinter diesem didaktischen Würfel ist nicht, eine Inhaltsstruktur des globalen Lernens zu entwickeln, da entsprechende, Themen' erstens schon in vielfältiger Form z.B. in Empfehlungen, Lehrplänen, Schulbüchern und Unterrichtsmaterialien vorliegen, und da zweitens das Themenspektrum des globalen Lernens nicht nach einem Kanonprinzip, sondern als prinzipiell offen und unabgeschlossen gedacht wird. Drittens ersetzen eine Themenliste oder ein Katalog möglicher Ziele und Inhalte keinesfalls die Aufgaben didaktischer Analyse und Unterrichtsvorbereitung, die darin bestehen, Unterricht so vorzuplanen. dass er bestimmten inhaltlich-intentionalen Ansprüchen an globales Lernen genügt. 
Mit den hier vorgelegten didaktischen Strukturierungskriterien kann - so die Idee - zweierlei geleistet werden: Der didaktische Würfel zur Strukturierung des globalen Lernens dient einerseits als ein kritisches Analyseinstrument zum Aufspüren von Einseitigkeiten, Defiziten und Verzerrungen in vorliegenden Curricula, d.h. in Programmen und Projektpaketen, in Lehrplänen, Schuibüchern und anderen Unterrichtsmaterialien, die in vielfältiger Form von staatlichen und nicht-staatlichen Instanzen als Bildungsmedien offeriert werden. Er kann andererseits von Lehrplankommissionen, Aktionsgruppen, Schulbuchautoren und Lehrpersonen als ein konstruktives Analyseinstrument zur Konzipierung neuer Curricula verwendet werden, d.h. zur Ausarbeitung von Unterrichtsmaterialien und zur Vorbereitung des Unterrichts

Der didaktische Würfel enthält in visualisierter Form drei miteinander verschränkte Gedankengänge (Dimensionen):

1. Die Achse ,Weitergabe von Kultur ' bezieht sich auf den Implikationszusammenhang von Zielen und Inhalten. Um dem ,globalen' Anspruch des Lernbereichs gerecht zu werden, muss ein Thema so aufbereitet werden, dass es sowohl Wissen vermittelt als auch zur Gewissensbildung beiträgt (scientia und conscientia). Dies soll dadurch geschehen, dass die unterrichtliche Bearbeitung des Themas normative, empirische und handlungsleitende Aspekte einschließt. Wenn z.B. ein Schulbuch eine Statistik über Armut in der Welt enthält (sprich: empirische Daten) und sonst, außer vielleicht einigen allgemeinen Arbeitsaufträgen, nichts, so würde dies als , defizitär ' diagnostizierbar und müßte um explizit normative Fragen und solchen nach möglichen Handlungsoptionen ergänzt werden. Wenn andersherum z.B. nur Projekte zur Armutsbekämpfung in einem Textheft vorgestellt würden, müssten die empirischen und die normativen Implikationen zur Sprache gebracht werden.

2. ,Kontextualität' macht darauf aufmerksam, dass dem Anspruch globalen Lernens nur dann genüge getan werden kann, wenn die Einbeziehung und die Wechselwirkungen verschiedener sozialräumlicher Kontexte vom Einzelnen bis zur Weltgesellschaft reflektiert werden. Die im Modell genannten drei Aspekte sind nur als Referenzbeispiele einer auch stärker differenzierbaren Skala gedacht, die vom einzelnen Individuum über lokale zu mikroregionalen, nationalen, makroregionalen bis hin zu globalen Betrachtungsweisen reicht. Wenn das Thema im Geographieunterricht beispielsweise das Amazonasbecken ist, darf sich der Unterricht, will er zu globalem Lernen beitragen, nicht auf die Geographie dieser Region beschränken, sondern muss den Horizont bildlich gesprochen - nach beiden Seiten der Skala hin ausweiten: auf Selbstreflexion hin (z.B.: Inwiefern betrifft mich das Amazonasbecken z.B. als Kon- sument von Möbelholz?) und auf die weltweiten Auswirkungen hin (z.B. ökologische Auswirkungen für das globale Klima).

3. Die ,historische' Achse gibt dem vermittelten Wissen und seiner unterrichtlichen Bearbeitung eine prozessorientierte Perspektive. Vergangenheit, Gegenwart und Zukunft bzw. ,Zukunft' im Plural gedacht als verschiedene Handlungsoptionen, sind vielfach miteinander verwoben. Rekonstruktionen geschichtlicher Ereignisse und Prozesse reflektieren unsere heutigen Bedürfnisse; die Deutung von Gegenwart enthält Visionen erhoffter und befürchteter Entwicklungen. Die Beachtung dieser didaktischen Dimension soll gewährleisten, dass Geschichte einschließlich der Zukunft als von Menschen gemacht in den Blick kommt. Unterrichtsmaterialien und didaktische Planungen unter dem Anspruch globalen Lernens müssen gewährleisten, dass ein Unterrichtsgegenstand im Sinne prozessorientierter Analysen von den Ursprüngen heutiger Entwicklungen bis hin zu den möglichen Auswirkungen derzeitiger Entscheidungen in den Blick kommt

Das didaktische Modell in Form eines Würfels vermag in besonderer Weise zu veranschaulichen, dass die genannten drei Dimensionen aufeinander bezogen sind und ein Ganzes bilden, d.h. ein Netz von miteinander verwobenen Aspekten, die alle zusammengenommen, globales Lernen' befördern sollen. Die didaktische Analyse und Unterrichtsplanung müssen gewährleisten, dass statt einer eher zufälligen, nicht nachvollziehbaren Amalgamierung von Wissensbeständen im Prinzip die gesamte Komplexität des ,Themas" im Unterricht in der gemeinsamen Arbeit von Lehrpersonen und Lernenden zur Sprache kommt. Mit Bezug auf die Referenzpunkte des didaktischen Würfels wird es z.B. möglich, begrïndet und kriterienbezogen anzugeben, warum und was im Unterricht passiert, wenn eine Statistik interpretiert oder eine Definition diskutiert wird, wenn Schüler Fakten lernen oder ein Planspiel durchführen. Eine Statistik über Kindersterblichkeit in Afrika wird z.B. durch die Koordinaten ,empirisch", ,regional' und , gegenwärtig symbolisiert und würde didaktisch dazu auffordern, die jeweiligen anderen Koordinaten im weiteren Unterrichtsverlauf mitzubedenken und durch entsprechende Fragestellungen, Arbeitsaufträge, Texte oder Tabellen zu repräsentieren. Dies müßte die didaktische Planung z.B. auf Vergleiche historischer Art oder auf normative und handlungsorientierte Gesichtspunkte lenken. In diesem Sinne versucht das Modell, den Inhalten und Zielen des globalen Lernens, die in Empfehlungen supranationaler Organisationen (z.B. der UNESCO), in Lehrplänen und Schulbüchern oder in Projekt- und Unterrichtsmaterialien verschiedenster Organisationen angeboten werden, ihre ,globale', unverkürzte didaktische Perspektive zu geben. Das Modell würde dann in beiderlei Hinsicht angewendet: in analytischer zum Auffinden und zur didaktisch begründeten Kritik von Defiziten in vorliegen- 
den Curricula und in konstruktiver Hinsicht zur Anleitung von Unterricht, der dem Anliegen globaler Multiperspektivität verpflichtet ist.

\section{Anmerkungen}

1 Die Entstehung und Verbreitung sowie Merkmale eines abkürzend als, Weltmodell Schule' bezeichneten Typus international zunehmend konvergenter öffentlicher Pflichtschulsysteme ist von der Autorin andernorts ausführlich dargestellt worden (Adick 1988, 1992, 1995, 2000). Die Existenz strukturell ähnlicher Schulsysteme weltweit ist Begleiterscheinung und Voraussetzung für Globalisierung. Ohne ein solches, Weltmodell Schule' wären, z.B. allein mangels Kompatibilität, Wissens- und Technologietransfer, ein Studium im Ausland, ein internationaler Arbeitsmarkt, aber auch Faktoren wie brain drain oder die Einflussnahme internationaler Organisationen auf nationale Bildungspolitik nicht denkbar.

2 Das Modell wurde erstmals vorgestellt in meiner Veröffentlichung zum Seminar, Curricula issues on living together', das unter der Leitung des Internationalen Erziehungsbüros (IBE) in Genf stattfand und zur Vorbereitung des 46 . Weltbildungskongresses der UNESCO (September 2001) einberufen wurde (Adick 2002, Kap. 2.3 u. 2.4)

\section{Literatur}

Adick, C./Bonne, L./Menck, P.: Didaktik des Pädagogikunterrichts. Stuttgart 1978.

Adick, C.: Schule im modernen Weltsystem. Ein Versuch zur Entmythologisierung der Vorstellung von Schule als Kolonialerbe. In: Zeitschrift für Kulturaustausch 38 (1988), S. 343-355.

Adick, C.: Die Universalisierung der modernen Schule. Eine theoretische Problemskizze zur Erklärung der weltweiten Verbreitung der modernen Schule in den letzten 200 Jahren mit Fallstudien zu Westafrika. Paderborn 1992.

Adick, C.: Internalisierung von Schule und Schulforschung. In: Rolff, H.-G. (Hg.): Zukunftsfelder von Schulforschung. Weinheim 1995, S. 157-180.

Adick, C.: Globalisierung als Herausforderung für nationalstaatliche Pflichtschulsysteme. In: Scheunpflug, A./Hirsch, K. (Hg.): Globalisierung als Herausforderung für die Pädagogik. Frankfurt a. M. 2000, S. 156-168.

Adick, C.: Didactics of global education: a tool for combining the international and intercultural dimensions of education. In: Vivre ensemble et éducation. Actes du colloque "La problématique du vivre ensemble dans les curricula», hg. v. F. Audigier/N. Bottani. Cahier du SRED (Service de la recherche en éducation) No. 9, Genf 2002 (im Druck).

Blankertz, H.: Theorien und Modelle der Didaktik, München 6. überarb. Auflage 1972.
BLK (Bund-Länder-Kommission für Bildungsplanung und Forschungsförderung): Bildung für eine nachhaltige Entwicklung - Orientierungsrahmen (Materialien zur Bildungsplanung und Forschungsförderung, H. 69), BLK Bonn 1998.

BLK (Bund-Länder-Kommission für Bildungsplanung und Forschungsförderung) u.a.: 66 Gute Praxisbeispiele zur Bildung für eine nachhaltige Entwicklung. BLK-Kongress 2001 in Osnabrück. CD-ROM.

Butterwegge, Ch./Hentges, G. (Hg.): Politische Bildung und Globalisierung. Opladen 2002

Davies. S./Guppy, N.: Globalization and Educational Reforms in Anglo-American Democracies. In: Comparative Education Review, 41 (1997), H. 4. S. 435-459.

de Haan, G./Seitz, K.: Kriterien für die Umsetzung eines internationalen Bildungsauftrages Bildung für nachhaltige Entwicklung. In: 21 - Das Leben gestalten (Zeitschrift. hg. von der Koordinierungsstelle des BLK-Programms 21), Teil 1: H. 1 (Jan. 2001): Teil 2: H. 2 (April 2001).

Deutscher Bundestag: Beschlussempfehlungen und Bericht des Ausschusses fïr Bildung. Forschung und Technikfolgenabschätzung. Bildung für eine nachhaltige Entwicklung. Drucksache 14/3319 vom 10.05.2000.

KMK (Kultusministerkonferenz): Empfehlung zur Förderung der Menschenrechtserziehung in der Schule. Beschluß vom 4. Dezember 1980 i.d.F. vom 14. Dezember 2000.

KMK (Kultusministerkonferenz): Empfehlung zur Interkulturellen Bildung und Erziehung in der Schule. Beschluss vom 25. Oktober 1996.

KMK (Kultusministerkonferenz): Empfehlung zu „Eine Welt/Dritte Welt“ in Unterricht und Schule. Beschluss vom 20. Februar 1997.

Menck, P.: Unterrichtsanalyse und didaktische Konstruktion. Frankfurt a. M. 1975.

Menck, P.: Looking into Classrooms: Papers on Didactics. Stamford/Connecticut 2000.

Menzel, U.: Globalisierung versus Fragmentierung. Frankfurt 1998.

Posch, P./Larcher. D./Altrichter, H.: Curriculum/Lehrplan. In: Taschenbuch der Piidagogik hg. v. H. Hierdeis/Th. Hug. Hohengehren 4. vollst. überarbeitete und erw. Auflage 1996, Bd. 1, S. 184-202.

Scheunpflug, A./Hirsch, K. (Hg.): Globalisierung als Herausforderung für die Pädagogik Frankfurt a. M. 2000.

Schöfthaler, T.: Vom additiven Wertkonsens zur Bildung für das 21. Jahrhundert. Die internationale Entstehungsgeschichte des Globalen Lemens. In: Zeitschrift für internationale Bildungsforschung und Entwicklungspädagogik. 23. Jg. (2000). 3. S. 19-23.

Seitz, K.: Lernen für ein globales Zeitalter. Zur Neuorientierung der politischen Bildung in der postnationalen Konstellation. In: Butterwegge, Ch./Hentges. G. (Hg.): Politische Bildung und Globalisierung. Opladen 2002, S. 45-57.

Selby, D.: Global Education as Transformative Education. In: Zeitschrift für internationale Bildungsforschung und Entwicklungspädagogik, 23. Jg. (2000), 3. S. 2-10.

Stromquist. N. P./Monkman. K. (Hg.): Globalization and Education. Integration and Contestation Across Cultures. Lanham etc. 2000

Tye. K. A.: Global Education. A Worldwide Movement. Orange/California 1999. 
UNESCO: Recommendation concerning education for international understanding, co-operation and peace and education relating to human rights and fundamental freedoms. Adopted by the General Conference, Nov. 1974.

UNESCO: All human beings ... Manual for human rights education, Paris 1998

VENRO (Verband Entwicklungspolitik Deutscher Nicht-Regierungsorganisationen):

Bildung 21 - Lernen für eine gerechte und zukunftsfähige Entwicklung. Kongressdokumentation. Bonn 2001.

Weinbrenner, B./Fritzsche, B.: Teaching Human Rights: Suggestions for Teaching Guidelines, hg. v. der Deutschen Unesco-Kommission, Bonn, und dem Georg-Eckert-Institut für Internationale Schulbuchforschung, Braunschweig, 1993.

WUS (World University Service - Deutsches Komitee): Globales Lernen/Bildung für nachhaltige Entwicklung. Engagement für die schulische Bildungsarbeit in der Bundesrepublik Deutschland. Wiesbaden 1998.

\section{Kurzbiographie}

Christel Adick, Dr. phil. habil., Dipl.-Päd., seit 1993 Lehrstuhlinhaberin für Vergleichende Erziehungswissenschaft am Institut für Pädagogik der Ruhr-Universität Bochum. Forschungs- und Lehrerfahrungen an der Westfälischen Wilhelms-Universität Münster, der Universität-Gesamthochschule Siegen, der Humboldt-Universität zu Berlin und der University of the West Indies, Cave Hill Campus (Barbados).

Anschrift: Institut für Pädagogik, Ruhr-Universität Bochum, 44780 Bochum;

E-Mail: christel.adick@ruhr-uni-bochum.de; www.ruhr-uni-bochum.de/ve 